UDC 513.72

SCOPUS CODE 1802

https://doi.org/10.36073/1512-0996-2021-1-119-135

\title{
Control in the Space of Life
}

Sergo Dadunashvili

\author{
Department of Electrical Engineering and Electronics, Georgian Technical University, \\ Georgia, 0160, Tbilisi, Kostava Str. 75 \\ E-mail: dadu@gtu.ge
}

\section{Reviewers:}

G. Dgebuadze, Professor, Faculty of Power Engineering and Telecommunication, GTU

E-mail: project7@gtu.ge

K. Kamkamidze, Professor, Faculty of Informatics and Computer systems, GTU

E-mail: kkamkamidze@yahoo.com

\begin{abstract}
Life manifests itself as a purposeful positive selection. Living, as a procedural, unmanageable itself to the usual methods of analysis. The answer to the question of how life is arranged and what a living system is based on in reality is an urgent task.

The article discusses the general principles of work and the manifestation of dual relations in the space of life, taking into account cosmo-physical and geophysical factors, using the example of the relationship between the virus and the cell.
\end{abstract}

Key words: cell; duality; relationship; structureless control; virus.

\section{Introduction}

An outbreak of pandemics is not just an accident, but a coincidence, it is always a unique situation that is caused as a result of a change in the state of the environment, in which the organism finds itself in new conditions of interaction with other living beings. There are a lot of viruses around a man, and they are much older than mankind. It often happens that viruses are looking for a new field of activity and become dangerous for man. When a virus changes its natural habitat, crosses the interspecies barrier, and finds a man, many problems and risks arise.

How does the virus work? It doesn't feed, it doesn't generate energy. The virus does not multiply by division like cellular organisms. It does nothing at all, except for replicating. The virus uses the cells of the organism it has entered, if it is specific and dual to these cells. Each virus has its own characteristic, which it uses to embed into the cells of a living organism, and this interaction determines what the infection will lead to. How a virus is protected globally and locally depends on its nature and how the virus spreads. First, it is transferred to the living cell, attaches to it, and then is embedded in the cell. When the genomic nucleic acid of the virus is "undressed" from the envelope, replication of the viruses begins through protein synthesis. The cell repeatedly reproduces the genome of the virus and, in parallel, the protein for the envelopes of the virus copies. When new genomes "dress" in their protein, replicated viruses are 
released into the intercellular space and the original cell most often dies.

If the virus is not cell specific, then embedding will not occur. Why do cells show philia and love, and begin to reproduce a virus? This is how duality works. The cell has its own genome. It acts as a scanner. If a fragment of specified external genetic material gets into the genome's area, the cell will try to embed it into its genome. After all, if she survives, she may be more perfect. For example, a cell can process some chemical element that has not yet been absorbed, produce a new enzyme, and so on. When viruses embed their genetic material into the genome of host cells, the cell may acquire some new qualities, or not acquire, and even die. Nevertheless, cells always try to seize the genetic material they meet so that a more updated and perfect genome is transmitted to the offspring, which provides program-genetic control to her existence. Consideration of the described phenomena as dynamics of the dual relationship < virus (V) |cell (C)> allows obtaining a number of new results.

\section{Main part}

\section{Structureless Control}

How is life arranged and what is the basis of a living system in reality? Life, like a stream, does not give in to usual receptions of the analysis. In the biosphere, life is the way nucleic acids exist. One of the variants of existence of nucleic acids is viruses, which need other organisms for life. They do not care at all about their well-being, but only try to adapt to their environment, like all organisms on the planet.

Negative selection is the controlling mechanism of life's activity. Adaptation acts as the biological equivalent of proto-intelligence. The role of the environment is to preserve or destroy life. This excludes the absolute internal causality of life, which cannot be reduced to external causes. The task is to achieve high accuracy of control over the change in the parameters of the internal and external environment of a living system. Everything that happens around is the diversity and complexity of its manifestations, its transformation of forms, and its renewal by the generation of itself, and all metamorphoses are observable, but invisible to ordinary vision.

The main principle that determines the functioning of life at any level is the competitive interaction of autonomous, uncorrelated actors - members of a certain population. This is the principle underlying structureless control. Its algorithm is realized without use of an external organizational structure. Working tools in structureless control are created based on such factors and technologies that can influence participants through activity patterns, stereotyping, fear, pressure, use of natural needs, etc. Technologies for structureless control of elements without an external structure, i.e. not bound by any obligations and not receiving directive commands and not knowing each other until the moment of control.

Initially, extreme (polarized objects) are cultivated, which are in tough opposition. This process has a special quality, all who are in front of the process are involved in it without knowing it. Thus, objects are involved in certain events, as a rule, these events are informationally significant for object. Further, an address-less information module (virus) is launched for everyone who directly perceives the reality, has a direct reflection of information that fits into the real-time scale. This is certain information that elements pick up and transmit further beyond the conditional boundaries of coverage. Elements pick up and convey information because of their interests and the interests of its group.

The term "super-system" has been chosen to indicate a system that contains a multitude of embedded system elements. If the spread of the launched information module is predictable, then a nonzero probability may arise that a certain number of elements of the supersystem based on their own self-control, but under the influence of the distributed information module, will themselves fold into structures.

1. The number of structures can be from one to many, but each of the structures will carry a control algorithm that works to achieve the goals associated with the launched information module.

2. The goals associated with the launched information module will be achieved, but the structures that will ensure the achievement of these goals will be formed in 
the process of structureless control and after they are formed, each of them will begin to work in the already structured control mode.

3. The established structures can compete, some of the structures may fail to achieve the goal, some of them will achieve the goal, but one way or another the structureless control, started with the help of the information module in the probabilistic and statistical sense, will lead to the goal.

4. Structureless control is built on the basis of the information that is already present in the memory of at least a certain proportion of the elements that make up the super-system in which the information module will be distributed.

5. If there is no information and algorithmic stereotypes for the response of elements included in the supersystem for the actions planned in the information module, then the information module simply will not find a response.

6. Structureless control begins immediately if there are response stereotypes in the memory of the elements, which allow the implementation of the appropriate algorithm and achieve the goal, adequate to the information disseminated by the module.

The guide for the progress of the process is what is commonly called the "information environment", the information infrastructure in which the current data of the elements is exchanged.

In the process of control, the closed system and its part - the control system - form a structure determined by the vector of goals and bearing the concept of control as a component of its objective function. The quality of control is ensured by two factors:

the architecture of the structure, i.e. functional load of its elements (including data exchange channels) and ordering (organization, hierarchy) of elements in the structure;

performance characteristics, functional suitability of the elements included in the structure for the implementation of the functions assigned to them (the level of complexity of the elements).

Structureless control is possible in super-systems, which consist of many similar, in a sense, elements to each other. Each of the elements of the super-system has the ability to memorize information passing through it and transmit information in a probabilistic manner to other elements included in this set; that is, the set can have forward and backward mapping processes. The behavior of the elements of this set is determined by their internal informational-algorithmic state.

Circular dissemination of information (that is, the same information passes through many elements), subject to certain statistical characteristics and various assessments of the possible course of events, carries a probabilistic predetermination of changes in the information state of the memory of the elements of the set. Such a change leads to a change in the statistical characteristics of the self-government of the elements.

If the dissemination of information in this set and its consequences have stable predictability in the statistical sense (that is, it generates predictable statistics of phenomena), then it is possible to manage this set without structure, as well as its structureless self-control. In such a set of elements that have different informational states of their memory, subject to statistical laws, there is a probabilistic predetermination and the likelihood that circular unaddressed passage of a certain content in the environment of an information module will lead to the fact that the elements of the set, on the basis of selfmanagement, will fold into one or more structures, focused on a certain vector of goals corresponding to the specified information module during a completely acceptable time interval. In other words, in case of structureless control, a set of more or less similar elements to one another in a probabilistically predetermined manner generates closed systems that correspond to a given vector of goals and a set of admissible error vectors. [1;2]

In case of structureless control, the structure is not formed in a directive-targeted manner before the start of the control process, but arises in a controlled and probabilistically predetermined manner in the course of the control process on the basis of a predominantly address less circular dissemination of information. Therefore, the set of elements in which the process of structureless control takes place is itself a closed system 
of hierarchically ordered loops of forward and reverse links, the architecture of which changes during the process of managing the environment, which generates structures from itself in the process of its self-control.

Structureless control in its essence is the control of statistical characteristics of multiple (mass) phenomena on the basis of probabilistic predeterminations of storage, dissemination and processing of information dominating over a set of elements and their estimates based on measures and statistical models. If the duration of the process exceeds the maximum lifetime of the elements of the system, then a stable matrix (frame), "staffing table" appears in the system, which is filled with updated elements as necessary.

The objective basis of structureless control is statistical predetermination and probabilistic models (subjective assessments of objective statistical predeterminations), which order mass phenomena in a statistical sense, allowing to distinguish one statistic from another; and in many cases, identify the reasons that caused the difference in statistics. The value of the probability, the statistical frequency, as well as their various estimates are measures of the control uncertainty. They are also measuring of the stability of the transition process leading from a certain state (possibly identified with the present) to each of the various options for the future in the set of possible.

\section{Language of Nature}

The single language of Nature is the code of existence, in which actors interact and comment on reality. The activity of actors is largely reduced to competition, when the actor produces his own codes (languages, terminology, ways of expression) and absorbs or crushes others, turning them into uninteresting for an external observer - and ultimately - collapsing. Competition of codes: the death of the subject, actor, and individuality shifts the emphasis to small groups that have their own language (code) and their own interpretations of the environment. The small group is capable of collective action. Competition between small groups and their languages (forms of expression, methods of interpretation) occurs within the framework of the control system. Bilateral regimes are emerging and multilateral formats are being implemented.

The life of nature is a language; an event in nature is a text; and the meaning of the text is interpreted based on the context. Context creates the dual relationships DR, which, have integrity, inextricably combining (utility, meaning and harmony) and claiming to harmonize Nature. The system $\langle\mathrm{V} \mid \mathrm{C}\rangle$ component ambivalence represents as a whole a universal object (UO). This object brings its data, its own rules of the game and its information models into the "information environment" (space), the information infrastructure in which the current data of the elements is exchanged as resources. Reading from this medium determines the embedding at time. The distinction between the "visible" and "significant" part of events creates their categorical distinction into constant and virtual, where virtuality is a new type of event which does not disappear. In UO the language of nature gives one of its finished products. From event to event, from image to image, the content of the product unfolds as a connection of succession in time and is represented as an arranged panorama in space. No nature was made up of systems $\langle\mathrm{V} \mid \mathrm{C}\rangle$, but, on the contrary, the system $\langle\mathrm{V}| \mathrm{C}>$ originated from the organic totality of the language of nature.

Control implemented in the system $\langle\mathrm{V} \mid \mathrm{C}\rangle$ (see Fig. 1) includes procedure determined by the control code. Code manages living systems (LS) and defines their behavior. The alphabet of the code serves as the basis of the system. These foundations - plan "Y" codes, define the keys to understanding the plans of the considered circuit. The code matrix is used to transfer information between plans.

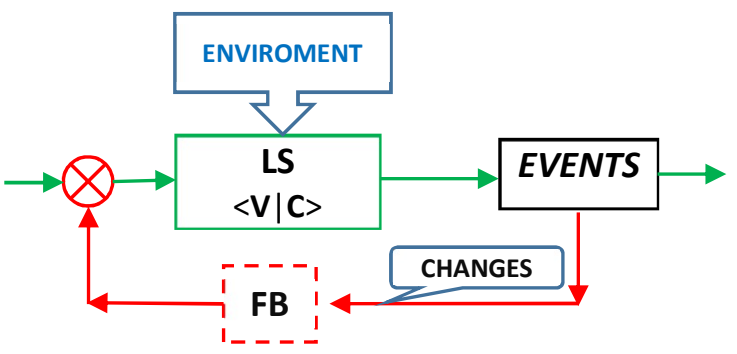

Figure 1. Control in DR 
Relationships <cell demand - virus supply $>$ form their own "Creation Field". Between the levels there is a "Potential of Creation" of relationship and there is a hunt for resources. This "procedural" formation is formed due to the "splitting" of the limit of field-forming relations, and is no longer characterized by external, but by internal duality. The determining factor is the "internal" dynamics. In the formula $(\Phi)$, the left side characterizes the properties of supply and demand, and the properties of relations on the right side, in relation to the properties of the left side, are characterized by negative dimensions. This is the relationship of the "external" and "internal", they are, as it were, "turned inside out" in relation to each other. In the interrelation of adjacent levels, when the inner is reborn into the outer, an entry into the field occurs. Not all complexes can move to "action" and remain at the stage of "striving". Of the emerging aspiration complexes, those that are in accordance with the goal complex are retained and developed to the degree of "action". The ability for conscious systemic effects is manifested as direct operation.

"Supply-demand" is self-regulation engine of DR. There are demand reactions and supply reactions. The pressure causes reformatting, reallocation of resources and operation. The imbalance represents a call to action and begins to emerge from the state of inner balance. When the balance <demand-supply $>$ is violated, starts the search for the positions of the poles in the relation corresponding to stability, i.e. balance of operations.

Behind the "procedural" are the patterns of demand and supply. There is no growth without demand. Each of the relations of demand and supply is already initially characterized by internal duality. However, the relationship between demand and supply is also to control the "procedures" of a higher level of the hierarchy. It is this level that determines the Design (the rules of the game) that generates all relationships.

Intelligent Natural language (notional, understandable language) needs an alphabet as a hold and a wordbuilder. This is the deepest, most stable and most essential structure of existence, eternal and universal necessity. This is the multiplicity that always remains equal to itself. Action of the alphabet absolutely based on movement, transforms blind elements into intelligent, in the form of a consistently unfolding presentation. The alphabet is both the content of thought and the expression of thought. It is both in activity and in the manifestation of intelligent at the same time.

The alphabet belongs to the nature, but not to the subject and serves to preserve the stability and harmony of the world. It defines the totality of form-creating potencies (seed), but it will not contain the power. The alphabet forms the code of the intelligence, thanks to which the system of the subject is included in the system of the Universe. In this case, the alphabet indirectly serves the essence of the subject. The alphabet serves as a "mediator" as it determines what is sent and manifested in the intelligence. What does it mean "to present this foundation, this sense of reality, to yourself and to others?" - It means to name it, i.e. give it a name and proclaim it. In this case, the activity and manifestation of intelligence are given in their unity. This event becomes inextricably linked with interpretation. The way of presentation is recognized as self-sufficient and self-valuable. The narrative unfolds "for the sake of the story itself," and not for the sake of direct impact on reality, that is, ultimately, outside of any function, except for symbolic activity as such. Life "by itself" does not exist: the main content of events is transferred into the process itself and the way of narration, and an idea of reality is formed.

\section{Virus and Cell in Nature}

Viruses are genetic formations consisting of one type of nucleic acid, which is protected by an envelope that also contains a combination of proteins, lipids and carbohydrates. A virus is a non-cellular agent that lives and can reproduce only inside the living cells of the host. It does not contain the most complex element of living systems - the translation apparatus (protein synthesis), the degree of complexity of which exceeds the intrinsic complexity of the virus.

Viruses are the oldest biological agents on Earth. There are many types of viruses in the world that can infect almost all types of living cells, while the hosts are 
all types of organisms, starting with unicellular, bacteria, fungi, plants and ending with animals and humans. However, different types of viruses can infect only a limited range of hosts.

Viruses are a natural facility (container) for the transfer of genes between different types of organisms, which creates genetic diversity and guides evolution in Nature. They play an important systemic role in the regulation of the population of living organisms. The spread and transmission of viruses is provided by a variety of carriers, including natural elements and living organisms. Viruses are believed to have played a crucial role in early evolution to trigger the differentiation process, during the time of the last universal common ancestor of life on Earth. As a result, viruses have genetic links with most of the earth's flora and fauna.

The main role of viruses in the biosphere is associated with their activity in the waters of the oceans and seas. The number of viruses in ocean bottom sediments is practically independent of depth and is very high everywhere. The abundance of ocean viruses has a tremendous impact on life in Nature. The strength of ocean viruses is their high potential for infection: every second trillions of ocean viruses find their carriers. Viruses kill about half of all bacteria in the oceans every day. Their killer efficiency keeps the reproduction of their carriers under control, and this serves as the basis for optimizing the Earth's geosphere and biosphere, since bacteria are the main geoengineers of the Earth. When bacteria that fall prey to viruses die, their shell breaks, and they release billions of tons of carbon. Part of the released carbon stimulates the growth of other bacteria, while the other part settles on the ocean floor. For millions of years this "snow" of dead bacteria affects the temperature of the planet. Moreover, dead organics tend to turn into minerals.

Oceanic viruses are striking not only in their number, but also in their diversity. To date, over a million oceanic viral genes have been identified. Only $10 \%$ of these genes have analogs in the genome of bacteria, animals, plants, or even other viruses. The remaining $90 \%$ completely unknown to science. One of the prerequisites for the existence of such a variety of oceanic viruses is the abundance of carriers. Reproducing viruses, the host cell can add their own genes. Carrying the genes of the former carriers, viruses begin to introduce them, along with their own, into the genetic material of the new carriers. Sometimes "borrowed" genes make the carrier of the virus more adaptable in the process of development and reproduction. The success of the carrier automatically means the success of the virus.

Viruses demonstrate a huge number of variants of genome organization - the totality of hereditary material necessary for their construction, preservation and reproduction. According to this parameter, they are more diverse than other organisms. Viruses are the largest repository of genetic diversity on Earth. Gene circulation has had a huge impact on the existence of life on Earth. The genomes of all living organisms contain hundreds of thousands of genes carried by viruses. The oldest traces of viruses on Earth are the fossils of ocean bacteria. Viruses do not leave fossils, but they leave a mark in the genome of their carriers. These traces prove the existence of viruses for billions of years.

Since viruses possess some, but not all, properties of living things, the nature of viruses is characterized as "organisms on the border of wildlife". The process at the border can be represented as the interaction of a complex of organic molecules with a living object. Viruses are similar to living organisms in that they have their own set of genes capable of creating viruses similar to themselves and evolve by natural selection. However, viruses lack such important characteristics as cellular structure and their own metabolism, and it is these characteristics that are usually considered as the fundamental properties of living things.

Viruses are able to reproduce in special conditions, in the cell infected by them, the self-reproduction abilities of viral particles are actualized, which begin to create their own copies by self-assembly. Self-assembly is one of the manifestations of self-organization in Nature, which consists in the fact that molecules with a complex architecture are spontaneously assembled from prepared "building blocks" - simpler molecules. This process simulates the evolution of structures in nature. A variety 
of organic molecules are used as "building blocks" for self-assembly results in highly symmetrical structures.

The cell provides special conditions for the virus, and is a living multiplicity that provides a synergy of several processes in which one can distinguish between the cloning line, and the outflow as a passageway, and the isolation of the reservation or habitat. The resource base of the cell is one of the leading variables in its system dynamics, along with the structural organization and genetic potential. These variables have variability in terms of potential, size, and feature set. Multiplicity is manifested in the unity of all the processes occurring (metabolism, replication, multiplication, etc.). Such living multiplicity, in dual processes, demonstrate own intelligence. The concept of "unity" is not applicable to the concept of "system". Unity is not something observable from the outside. This is an intrinsic property of any system. A system is a flexible unity of its elements, each of which has its own irreplaceable and complementary function. If the system becomes obsolete, its future is impossible without the arrival of novelty. Coming newness transforms all old systems and goals and directs to the future.

Outside the host cells, viruses are in a completely inert state, but they have a set of instructions (a genetic program) necessary to re-enter the cell and, subjecting it to their instructions, make them produce identical copies. The evolutionary significance of viruses lies in the long-term storage of genetic information isolated from changes in the external environment, retaining the ability to read and reproduce when returning to the cellular environment.

Viruses exist in the form of a protein envelope that abduces the genetic material and protects this information carrier. The envelope is composed of identical protein subunits encoded by the viral genome, and its shape is the basis for the classification of viruses by morphological characteristics. The general form of the protective protein envelope of the virus is characterized by a high degree of symmetry, which determines the ability of viruses to crystallize. Some viruses have a welldefined icosahedral head and tail with spiral symmetry. Many viruses appear spherical, but retain icosahedral symmetry. A regular icosahedron is the optimal shape for a shell composed of identical subunits.

While the virus is in the extracellular environment or in the process of cell infection, it appears in the form of an independent particle that does not show signs of living and behaves like crystals of biopolymers. The simple structure, the absence of organelles and their own metabolism, allows some viruses to crystallize in the external environment, which is characteristic only of nonliving material. It was found that their crystals are composed of several hundred billion such particles closely pressed together. However, the reproduction of viruses differs from crystal growth in that viruses inherit mutations and are under the pressure of natural selection.

Depending on what type of nucleic acid is the genetic material inside the viral particles, the methods of its replication are also determined. Viruses differ in the localization of their replication, some viruses multiply in the cytoplasm of the cell, and some - in its nucleus. Viral genomes, regardless of the type of nucleic acid, are almost always either single-stranded or double-stranded. This determines the mechanism of genome replication. RNA viruses usually use the RNA nucleus as a template for the synthesis of viral genomic RNA and mRNA. The viral mRNA directs the host cell to synthesize viral enzymes, envelope proteins, and assemble new viruses.

An important factor in the case of a single stranded genome is its polarity. For most RNA viruses, the polarity of the nucleic acid is determined depending on whether it can directly serve as a template for protein synthesis. A (+RNA) molecule with positive polarity has the same nucleotide sequence as the mRNA, so it can be immediately translated by the host cell [3].

Genetic changes occur in viruses through different mechanisms. These include random substitutions of individual bases in genetic material. These point mutations are "silent" - they do not change the structure of the proteins encoded by the mutant genes, but sometimes, as a result of such changes, the virus can acquire evolutionary advantages, such as resistance to antiviral drugs. RNA viruses often exist as quasi species or an assembly of viruses of the same species, but with 
slightly different genome nucleotide sequences. Such quasi species are the main target for natural selection.

In the external environment, the genome of the virus is subject to various influences, for example, ultraviolet radiation, solar radiation, chemicals, which leads to various kinds of mutations, i.e. changes in the structure of the nucleic acid. Depending on the nature of the mutations, viruses can change their properties, and even change the host.

\section{Virus as a Nano-Object}

The nano range "borders" on the scale with the "micro" level (characteristic size - microns), which sets the "structure-sensitive" properties of objects; and with an atomic level that determines the quantum characteristics of matter. The synergy of the effects observed at each of these levels characterizes the nanoobject as a whole, at the macro level.

The nano range is characterized by the manifestation of properties that differ from the chemical, physical or biological properties of the macro state of a substance. In particular, the effects of quantization, tunneling, and a decrease in the Coulomb barrier are manifested in the nano range; the role of intermolecular interactions becomes significant. And also, the formation of regular nanostructures, in cells and viruses, capable of retaining and capturing quantum particles and enter into interaction with them.

Taking into account the above, consider the events in the space of life. Proto-intelligence is not introduced into nano-objects from the outside, but is a specialization of information systems that are originally characteristic of life. Information exchange is an integral component of the activity of self-regulating functional systems. It is in functional systems that there is an element for the qualitative and quantitative assessment of information an acceptor of the result of an action, which constantly analyzes and evaluates the state of useful adaptive results, to which the activity of various subsystems of the body is directed. "Unitary" reactions in the implementation of "intelligent activity" carried out by the "basic dynamic element" aimed at satisfying the leading biological or social needs of the organism. The properties of the "basic elements" fit corresponding DR \{need-satisfaction\}, \{cell demand $D_{c}$ - virus supply $S_{v}$ \}.

With a systematic approach, events in the information space present the following phases. Data on the needs of actors enter the information space as a "reference stream", and data on satisfaction of the need - as a "stream of resources". The interaction of the reference and resources streams on specific structures of space is carried out on an interference basis. An integral acceptor of the result of an action, organized by a multitude of actors - functional systems of a homeostatic, behavioral nature, acts as an information "screen".

Processes are formed by the universal object $\langle\mathrm{V} \mid \mathrm{C}\rangle$. Any changing of internal events in the virus and in the cell always has an external projection. Projection is manifested in the form of mass, quantity, density. As well as, through the redistribution of resources and actions, "compaction" and "exhaustion" of the space of objects.

Only together $\langle\mathrm{V} \mid \mathrm{C}\rangle$ forms a tandem, which quite adequately balances the entire power of the environmental impact, and provides a global balance. This form of registration of relations determines their uncertainty for competitors in the environment, the complexity and ambiguity for them of determining the actions of the parties to the pair. Competitors can be surrounded in a privileged position, produce internal separatism in the environment, tied to trans-dyadic structures. However, the stability of the tandem indicates the lack of systemic influence among competitors. The DR, while maintaining intelligence, achieves much larger and more stable results.

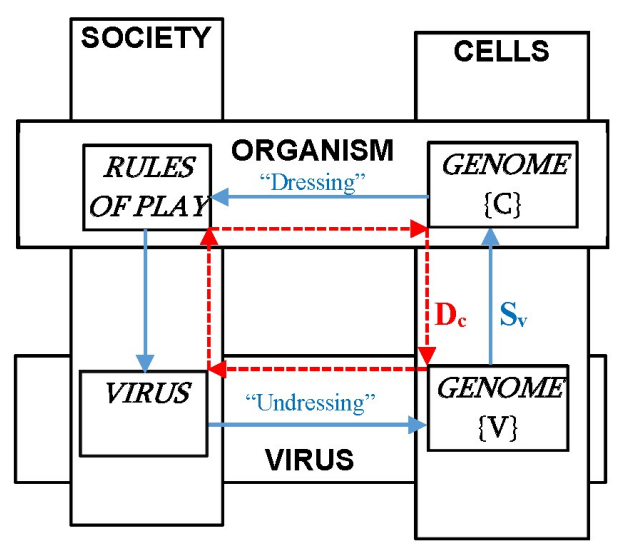

Fig. 2. RELATIONSHIP $<\mathrm{V} \mid \mathrm{C}>$ 
Figure 2 shows relationship $\langle\mathrm{V} \mid \mathrm{C}\rangle$. An object, virus, with a structured resource, manifests it explicitly. The subject, being structureless, carries a message (proposal) about this resource. This is how the coherence and unity of the structural and functional aspects of the relationship is manifested. Not from the benevolence of object, the subject demand is satisfied, but from observance by object of their own interests. Demand is directed not to humanism of object, but to its egoism. The subject informs it only on its benefits. What the object wants is received, when others, absolutely indifferent to its goals, want to receive their own benefit. In this case, its task is also "automatically" solved. This is one of the "automatic" wonders of the DR. Its result is using the population roles of actors, especially - the systemic role of the virus. The subject reflects the intelligent design of creation. The intention of the future creation contains belonging to the generic essence and determines the rules of play of process of materialization. The result of materialization can be alienated from the generic entity.

Content (V) is determined by its genotype. Each genotype is "settling" in $(\mathrm{V})$ the information process of coding information. In this intelligent dematerialization of oneself, a new genome is formed, which grows in the further process of self-assembly into itself. What is the function of content $(\mathrm{V})$ ? From outside the cell received, taken by the cell from the treasury of the natural language, someone else's creativity, in individual use it is created anew by the cell, again becoming in a state of origin and assembly of composition (V): fresh and renewed every time.

Form serves as a logical function of content. $(\mathrm{V})$ is an intelligent entity, for it carries with it - the idea of its being. The stability in it of the main type of its structure, not a sensual, but an intelligent invariant (carrying its own meaning), its form-forming individual principle, in its own way guiding information processes, remains equal to itself. Together with (V), the genome, when it enters a favorable environment, penetrates into some object of the surrounding space (cell), and makes in it the change that it is able to receive. $(\mathrm{V})$ is "reading" into the object with all characters of the code contained in the genome $(\mathrm{V})$, awakened to reproduction. The genome is an algorithm for an individual structure; according to its plan, a very different content is expressed. He predetermines new clones and outlines the ideal boundaries for their lives. (V) is an invariant, but this invariant itself is not reproduced.

The information space is formed by actors carrying information about the environment and responding adequately to external influences. The information space is formed in the unity of the structural and functional (non-local) aspects, thus forming a network of participants, in which "each actor is really connected with each". In the information space, there are natural operational mechanisms, which are based on the principles of the "assessment tool" of DR. In the case under consideration, the relationship $(<\mathrm{C}|\mathrm{V}\rangle)$, the following patterns take place:

Each virus already initially, at birth, shows duality. It "searches" for one single "half" of its, with which it then connects. That is why, in the process of functioning, it already initially shows "intelligent".

$(\mathrm{V})$ as the subject of the work conveys basic connections. These "connections as such" are so "general" that they turn out to be just as "insensible" and, as a consequence, are devoid of any value at level (X).

Such interaction on the physical plane gives rise to an invisible level $(\mathrm{Y})$, "non-sensory" connection and the presence of a channel for such a dual pair.

Non-material information space plays the role of an amphiphilic interface, which begins to work in both directions, connecting pairs $(<\mathrm{C}|\mathrm{V}\rangle)$ into single systemforming tools.

As soon as the chain of the operating mechanism of the instrument "closes" on itself, as a result of its normalization, an integral spatial image is created, which "in the image and likeness" forms the instruments of a fundamentally different level and purpose and, ultimately, a single information space is formed.

D Reason is a multilevel concept and at each level it is formed and functions "in the image and likeness", in accordance with the formula $(\Phi)$ of DR.

This image is not material and as whole is at a non-sensible level. Although it can manifest itself as 
various indirect registered phenomena in the process of formation and existence.

The image represents only an informational aspect, but it does not represent the functions that the actors of the process perform. As a signal, $(\mathrm{V})$ is a certain minimum energy of a physical order - (nano energy), in the general case the least effective, and in this case, it is so small that few people reckon with it as an action in the external world. Those, despite this insignificant physical appendage of its own, (V) cannot be considered a true reality and there is only a content taken in the abstract.

However, the matter is not only in the amount of energy, but also in its dependence on quality, order, form, i.e. in what form is this amount of energy presented. Introducing the concept of guiding signals, one can find even the smallest amounts of energy producing the most powerful actions and changing the whole reality in the direction set by this ordered signal.

Nano energy (V) can be directed precisely towards a certain action, and will produce it, despite its quantitative insignificance. $(\mathrm{V})$ is nano-object, there is an extremely developed object, its energy, very finely organized, having a definite and highly differentiated structure and, therefore, as the "energy of form", it has a large intensity.

The physical substrate $(\mathrm{V})$ contains a synergy of nano energies that are in constant transformation. This subject (V) can move in space as a whole and, while moving, always has its own content with it. With appropriate tuning and acceptance by the environment, modulations (V) can be understood by the receiving cell as complex natural signals.

The holistic organization of all this plexus of modulations $(\mathrm{V})$, its strictly outlined individuality, its nonrandomness, even in the smallest details, can be seen from the fact that the smallest change in the integral complex $(\mathrm{V})$, a change that is hardly taken into account by means of physical analysis, is immediately identified and is recognized by the cell.

Two-component system is a molecular-biological mechanism that allows cells to sense and respond to changes in various environmental parameters. Twocomponent systems are found in representatives of all three domains of life, and are encoded in the genomes of their representatives. Typically, the two-component system consists of a membrane-bound receptor that senses changes in the environment and a corresponding response regulator that provides a cellular response, mainly through differential expression of target genes.

Two-component systems carry out biological signaling through a response regulator (RR). Response regulators may consist of a single recipient domain, but are usually multi-domain proteins containing a recipient domain and an effector domain, often with gene-binding activity.

The receptor transmission modulator senses a change in the extracellular environment, it carries out a modulation response and transfers the signal carrier to a receiver in the cell. The corresponding RR further catalyzes the carrier transfer reaction in its recipient domain. As a result of this reaction, RR undergoes a conformational change that activates its effector domain, and it, in turn, triggers a cellular response to a signal, activating or repressing the expression of target genes

The existence of complex multicellular organisms is possible due to the coordination of the processes taking place in their cells. This coordination is based on intercellular communication and signal transmission within individual cells. Together, this enables one cell to control the behavior of the others.

Signaling is one of the most difficult areas in the study of cellular objects $[4 ; 5 ; 6]$. The term "signaling" refers to any process by which a cell converts one type of signal or stimulus to another. Signal transmission pathways, or signaling pathways, are often organized as signal cascades: the number of substances involved in signal transmission increases at each subsequent stage with distance from the original stimulus. Thus, even a relatively weak stimulus can elicit a significant response when the signal is amplified.

The intracellular signaling system consists of numerous interacting signaling pathways leading from receptors on the membrane to the cytoplasm, and further to the nucleus, where the work of the genetic apparatus is regulated, and then back to the cytoplasm, to the cell surface and the extracellular environment. This system consists of intercellular signaling molecules, receptors on the cell 
surface, cytoplasmic signaling cascades, transcription factors that control gene expression, and executive proteins that determine the cellular response.

Extracellular signaling molecules are recognized by receptors associated with membrane substances. Further, the activated substances are transferred to the intended targets of the dynamic action. All of these substances regulate numerous executive proteins and transcription factors that determine specific cell responses. Each type of cell has its own individual set of proteins that provide specific cell responses to various stimuli.

\section{Cosmo-Earth}

The influence of the "space climate" on Earth can manifest itself in a decrease in the intensity of the flow of ultraviolet radiation, on which the life of organisms, especially viruses and bacteria, depends. Taking into account the periodicity of the effect of near space on the habitat of living organisms, the effect of ionizing galactic radiation on the mutagenic potential of populations, it can be assumed that pandemics develop with a significant weakening of solar activity and corresponding changes in the modes of functioning of the geosphere, biosphere, and anthroposphere.

The direct and indirect mutagenic effect of the quiet sun on micro and nano organisms is confirmed by the observation that the formation of new strains of pathogens is timed to each next similar phase of weakening of solar activity. All acute respiratory viral infections are seasonal, they are referred to as "recurrent viral infections" and tend to recur. The strains return after a time interval to where they circulated before.

The temporal ordering and synchronization of cosmophysical factors and the biosphere makes it possible to obtain a general picture of cause-and-effect relationships leading to destructive pandemic processes. External influence originates in the depths of the galaxy, it controls the activity of the solar system and through the sun determines the functioning of all spheres of the Earth. Of particular interest are the mechanisms of energy and information transfer from near space, the receivers of which are living organisms, which, being elements of the biosphere, cause various fluctuations in Nature.
The links of this cosmic system are observable, their influence is measurable at the level of global processes. In a particular case of a pandemic, these are the integral parameters of the rate of mutations of pathogenic organisms and the rate of their reproduction, the changes of which reflect global processes. The challenge is to capture these connections and trace their origins and evolution.

Earth with an atmosphere is an open thermodynamic system. Moving in space along its orbit, it is in a constantly changing gravitational field of influence from other planets, as well as variable thermal and corpuscular influence from the Sun. In addition to external influences, one should take into account the reaction of the Earth itself to these influences. First of all, tectonic, volcanic and electromagnetic.

Until now, in the reasons for the change in the global climate of the Earth and the consequences of its change for the anthroposphere, neither the corpuscular energy of the Sun, nor the emission of hydrogen and methane from the Earth's interior were taken into account. Although both of these parameters are quantitatively comparable to the flow of energy coming to the Earth from the Sun. Heat flows from the Earth's interior are also not taken into account. In fact, the Earth appears as a dead asteroid, without volcanoes, earthquakes, without a magnetic field, without interaction with the "solar wind".

Under the influence of wave electromagnetic radiation from the Sun, atmospheric gas is heated, ionized, dissociated, etc. Thus, the wave radiation of the Sun determines the thermal regime of the atmosphere, its circulation and composition. In this case, the nature of atmospheric processes depends not only on the time of day, but also on the position of their localization, since the position affects the amount of received wave radiation.

The action of the energy brought by solar corpuscular streams - the "solar wind", also affects the circulation, dissociation and ionization of the atmosphere. But at the same time the solar wave radiation is constant, and the corpuscular streams are variable. With increasing latitude, solar wave radiation decreases, and the influence of corpuscular flows increases. Under the influence of corpuscular streams of the Sun, the conductivity in the lower 
atmosphere changes. This significantly affects the modes of thunderstorm formation, the formation of clouds and precipitation, as well as the weather in general.

If imagining the balance of energies, then in addition to the wave radiation of the Sun and corpuscular flows, the Earth's own energy is no less significant. Its generation is due to the processes taking place in the core of the Earth. As for the Earth's own energy, the magnitude of the heat flow from the Earth's interior to the surface of the continents is known today. In addition to the heat of the earth's interior, hydrogen and methane are constantly emitted into the Earth's atmosphere. In the presence of lightning discharges, both gases burn in the oxygen of the atmosphere, releasing heat. From the combustion of billions of tons of methane released annually through rift faults, the Earth's atmosphere receives energy quite comparable to the energy of the Sun entering the Earth.

The combustion of methane and hydrogen occurs in the atmosphere in the presence of lightning discharges, which in turn are activated due to the corpuscular radiation of the Sun. In the absence of lightning discharges, methane and hydrogen, escaping along rift faults, do not burn in the atmosphere, do not heat it additionally, and do not form carbon dioxide when burned in oxygen. But, rising into the stratosphere, they react with ozone and reduce its concentration, forming "ozone holes". If thunderstorm activity does not allow methane with hydrogen to rise into the stratosphere, then these gases, burning, form moisture and carbon dioxide. All this directly affects the biosphere and the mobility of living organisms, including dual ones.

Thus, the main actors of global climate change and the main architects of the biosphere are still the Earth and the Sun, since their heat and mass flows are not subject to man. This does not mean that a person is incapable of harming the environment.

The latest studies of geospheres indicate that their complex multifaceted shape was formed due to the growth of the inner iron core of the planet. Knowledge of the patterns of its structure and growth dynamics allows for a better understanding of the nature of the observed changes in the environment and the features of the functioning of the biosphere. The existence of huge crystals in the planet's body is manifested in the localization at the edges and tops of these polyhedrons of the centers of seismic and volcanic activity, centers of mobility of the atmosphere, water and land, and other anomalies $[7 ; 8]$.

The most distant part of the planet from the surface is a volume-centered cubic lattice consisting of iron modifications. Such elements of the Earth's cubic figure were found in the depths due to measurements of variations in the wave propagation speed during earthquakes, and it turned out that the diagonal of the cube is located along the axis of rotation of the globe. The impact of the tops of the cubic core on the properties of the outer shells of the Earth is traced in the form of geophysical anomalies. The cubic core leaves clear imprints in the mantle, visible on the earth's surface from volcanic eruptions. The symmetry of the cube is reflected many times in the outer shells of the planet and in the objects of the biosphere. The processes in the lattice created by the crystalline matrix are also manifested in the vertical migration of chemical elements along tectonic faults, which contributes to an increase in biological productivity in the ocean.

Signals of vertical links in the earth's geosystem have increased dramatically in recent decades. The formation of channels of these influences is associated with the icosahedral and dodecahedral syngony in explaining the Earth's infrastructure and the actions of similarity laws at different levels of matter organization. The reflection of the properties of the icosahedron and dodecahedron in the nature of the Earth is confirmed by facts that can only be explained by the projection and influence of the tops and faces of these figures on the earth's surface.

The instrumental role of the long-range order is determined by the fact that the inner polyhedrons are endowed with energy, which deforms the earth's crust and other shells, causing them to move. The manifestation of the crystalline properties of the inner core in the outer shells of the Earth helps to solve the problem of the causes of modern environmental changes. First of all, it focuses on the search for possible sources of additional energy. At the same time, it turns out that in 
recent decades, natural processes have intensified in all shells of the Earth.

The meaning of the search for order in nature, the identification of "correctness" is consonant with the ideas about the key role of symmetry in the picture of the world. Generalization of the facts leads to the understanding that the Earth, obeying the general law of nature, grows due to the crystallization of the core, and this process finds diverse manifestations in the outer shells. They are united by the observed increase in energy, in particular, manifested in an increase in the density of biomass, which defies explanation, if we proceed from the prevailing ideas. The geometry of the dodecahedron and its dual icosahedron is directly related to the "golden" ratio. The icosahedral type of symmetry was considered convenient by living beings. Based on the principle of symmetry in the interaction of the crystal and the environment, it can be assumed that the inner core of the planet is a growing crystal, which by its growth induces the same symmetry in the shells of the planet, including in the earth's crust and in the objects of the biosphere.

The supposed "engine" of the general planetary mechanism, which forms the symmetry of the crystal in the earth's crust, received comprehensive theoretical confirmation in the process of studying new achievements in crystallography. According to these data, the surface of a crystal nucleus already has its own potential, the range of which increases with the growth of the crystal faces and thereby increases the length of its own force field. For crystal growth, the participation of external forces is not necessary, the crystal itself is an active and main participant in the phenomenon, organizing the growth process and creating quasi-crystalline structures at a certain distance from the crystal surface in accordance with its symmetry.

The substance of the depths is embedded in the earth's crust by vertical flows. Substances of all shells of the Earth are strung on uniform radiuses, which "like a hedgehog" diverge from its center and come out to the surface in the form of nodes of the power frame. Part of the substance of the streams of the subcortical envelope penetrates into the earth's crust, and the bulk of each of the streams is closed on the envelope. Depth material penetrating into the earth's crust along the edges of the dodecahedron promotes the transformation of vertical pressures into horizontal displacements of crustal blocks in the directions from the edges of the dodecahedron (rift zones) to the edges of the icosahedron. The uplifts of the continental crust facilitate the movement of surface water flows, and with them the particles of matter (including living ones) in the same directions.

\section{Role of Iron in the Organism}

Just as the shape of the Earth's core induces its properties on its surface, so the iron contained in the core affects similar materials. In the organism, iron is essential microelement. Despite its low iron content, it plays a special role in maintaining its vital functions. The lack of iron in the body, like its excess, negatively affects many functions and the health of the body as a whole, as it leads to damage to all organs and tissues.

The organism is unable to produce iron. Iron enters the body through food. In food, iron is mainly in an oxidized state and is part of proteins and organic acids. But the absorption of ferrous iron is better, therefore, in the stomach, under the action of gastric juice, ferric iron is released from food and turns into ferrous iron. After ferrous iron enters the small intestine, it enters enterocytes (epithelial cells of the small intestine) and together with them into the body, where part of it is involved in many body processes and is constantly used, for example, for the synthesis of heme (part of hemoglobin), and the other a part is deposited in reserve. Iron metabolism in the body is an optimally organized process.

The human body normally contains about $4.5 \mathrm{~g}$ of iron, which is involved in the respiration process. Iron is part of about 100 different enzymes and therefore indirectly affects oxidation reactions, the immune response, energy balance, cholesterol metabolism, the synthesis of genetic material, etc.

The main functions of iron in the body are:

$>$ transport of oxygen from the lungs to organs and tissues, including the skin. The actor is an erythrocyte. The composition of the erythrocyte includes hemoglobin, iron in the composition of hemoglobin binds 
and transfers oxygen from the lungs to all cells of the body;

participation in the processes of hematopoiesis the bone marrow uses iron to synthesize hemoglobin, which is part of erythrocytes;

$>$ detoxification of the body - iron is necessary for the synthesis of liver enzymes involved in the destruction of toxins;

$>$ regulation of immunity and increasing the tone of the body - iron affects the composition of the blood, the level of leukocytes necessary to maintain immunity;

participation in the process of cell division - iron is a part of proteins and enzymes involved in the synthesis of genetic material;

synthesis of hormones - iron is necessary for the synthesis of thyroid hormones, which regulates the metabolism in the body;

providing cells with energy - iron delivers oxygen to the energy molecules of the protein.

Iron in the body is stored in depot molecules. The body creates a special microsystem - ferritin, which "collects" and deposits iron from the entire body, which, if necessary, spent on the needs of the body. Iron folds and packs in a certain pattern up to 4500 iron atoms in one ferritin molecule. The main function of this complex water-soluble protein complex is the binding and isolated temporary storage of iron inside cells in a dissolved and non-toxic form for the body. Ferritin is found in almost all cells, organs and tissues of the body and is a donor of iron in cells that need it. Its amount critically affects the state of the body.

The ferritin molecule consists of a central core, in which iron is packed, and a protein membrane surrounding it. The newly formed shell protein unfilled with iron is called apoferritin. When apoferritin is formed, its central cavity is subsequently loaded with ferrous ions. This iron concentrates and crystallizes into ferric hydroxide, which forms the mineral core.

Apoferritin is not a membrane protein. It serves as a marker of a person's biological age and indirectly acts as a tumor marker. To study the interaction of membrane proteins, it is first necessary to understand how nonmembrane proteins interact with each other, and apoferritin is a good object for research here, as well as a component for creating a vaccine against a wide range of viral diseases.

For a deeper understanding of the structural features of certain proteins, it is necessary not only to solve their structure, but also to understand how proteins interact with each other in solution and in the membrane. Integral proteins embedded in cell membranes are targets for many manufactured drugs. The study of such proteins is necessary to decipher their structure. Deciphering the structure, in turn, is necessary for understanding the structural and functional features of the work of proteins, studying their interactions with potential drug candidates, and selecting the most effective drugs.

Apoferritin ensures the absorption of iron in the intestine, as well as the deposition of iron in the body. Apoferritin also performs a catalytic function at the beginning of the process formation of a ferroxidase core inside the protein shell. The rate of absorption of iron by apoferritin depends on the initial amount of iron in the protein molecule. Apoferritin is involved in the removal of iron from the ferritin molecule. It is one of the vital proteins in any cell type.

The study of the interaction of membrane proteins in the membrane is impossible without a deep understanding of the processes occurring in the membrane itself, and first of all, understanding the behavior of lipids in different phase states and their interaction with each other. The study and search for conditions for the formation of lipid cubic phases is an integral and extremely important part of the work. Lipid cubic phases were studied to optimize the crystallization process as the most promising and promising conditions for the crystallization of proteins to date.

A search was made for lipids forming lipid cubic phases. The basic set of parameters of the system was determined, and also the quantitative determination of the lattice parameters in lipid cubic phases was carried out. Since lipids have hydrophobic and hydrophilic parts, they are capable of structure formation in water. An amphiphilic molecule was obtained. When amphiphilic objects are placed in an aquatic environment, 
depending on the lipid/water volume ratio, they are capable of forming several phases and characteristic types of structural organization, including inverted objects with a "turned inside out" structure. It should be noted that the phases and structural organization of amphiphilic objects depend both on the properties of the environment and on the properties of the objects immersed in it, as well as on the external influence on this integral complex.

\section{Conclusion}

Depending on the ratio of all these influences, various phase states of the complex were observed. The most interesting are the so-called lipid cubic phases forming a cubic lattice. In the experiment, with a certain structure, a local increase in the concentration of proteins in solution is observed. The study of crystallization processes in dynamics made it possible to quantify them and calculate the necessary ratios of parameters for the formation of crystal nucleus of both membrane and nonmembrane proteins.

The account of cosmo-physical and geophysical effects is determined by the model of falling flat monochromatic wave on disseminating center, which is a formed complex molecule, which, under the action of radiation, becomes a source of a scattered spherical wave. This wave, in a wide range of concentrations for the apoferritin protein, determines the adjacent (close) order corresponding to higher concentrations than the initial ones, which determines the appearance of an embryo in an aqueous solution [9;10].

The results obtained will serve as the basis for further experiments in the context of the global task of penetrating into the essence of the structural and functional organization of membrane proteins, the interaction of proteins and lipids in membranes. They allow moving from observing external manifestations to understanding the inner essence of the ongoing processes.

\section{References}

1. S. Dadunashvili. System Pattern of Consciousness Functioning - International Symposium on Biomedical Engineering and Medical Physics. Riga, Springer, IFMBE Proceedings 38, p. 5-10. (2012). (In English);

2. S. Dadunashvili. System formation models in biosphere and a noosphere. - Tbilisi. Proceedings of the Georgian Technical University, №3 (414), p. 224-232. (1997). (In English);

3. S. Dadunashvili. About the regulating mechanism in serial replication systems - Tbilisi. Proceedings of the Georgian Technical University, №4 (466), p. 42-51. (2007). (In English);

4. S. Dadunashvili. Bio signals Processing in the Structural Levels of Body - World Congress on Medical Physics and Biomedical Engineering. Beijing, Springer, IFMBE Proceedings Vol. 39, p. 581-584. (2012). (In English);

5. S. Dadunashvili. Becoming of Ubiquitous Sensors for Ubiquitous Healthcare. - World Congress on Medical physics \& biomedical Engineering. Toronto, Springer, IFMBE Proceedings, IUPESM 2015, Pages 1416-1418. (2015). (In English);

6. S. Dadunashvili. Multilevel Signal Processing for Biomedical Nanodevices. - Springer, IFMBE Proceedings Vol. 55, p. 329-331. (2016). (In English);

7. Al-Zoubi, N., Schönecker, S., Johansson B., Vitos L., Assessing the Exact Muffin-Tin Orbitals method for the Bain path of metals. Philosophical Magazine. 2017, 97(15): pp. 1243-1264. (In English);

8. Q. Tao, J. Lu, M. Dahlqvist, A. Mockute, S. Calder, A. Petruhins, R. Meshkian, O. Rivin, D. Potashnikov, E. Caspi, H. Shaked, A. Hoser, C. Opagiste, R. Galera, R. Salikhov, U. Wiedwald, C. Ritter, A. R. Wildes, B. Johansson, L. Hultman, M. Farle, M. W. Barsoum, J. Rosen, Atomically Layered and Ordered Rare-Earth iMAX Phases: A New Class of Magnetic Quaternary Compounds. Chemistry of Materials. 2019, Just Accepted Manuscript, DOI: 10.1021/ acs.chemmater.8b05298: pp. 1-16. (In English). 
9. Bar-On Yinon M., Flarnholz Avi, Phillips Rob, Milo Ron, SARS-CoV-2 (COVID-19) by the numbers. 2020. (https://bit.ly/2WOeN64).

10. Dadunashvili S. Medical Expert System with the Properties of Artificial Intelligence - World Congress on Medical Physics and Biomedical Engineering. Prague, Springer, IFMBE Proceedings Vol. 68/1, p. 315-319. (2018).

DC 513.72

SCOPUS CODE 1802

https://doi.org/10.36073/1512-0996-2021-1-119-135

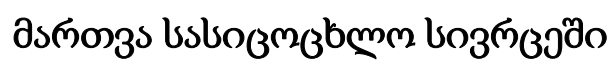

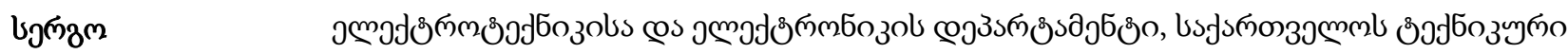

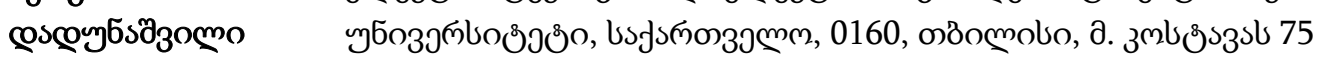

E-mail: dadu@gtu.ge

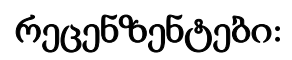

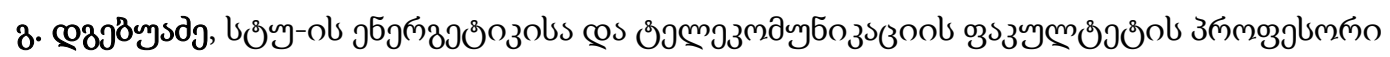

E-mail: project7@gtu.ge

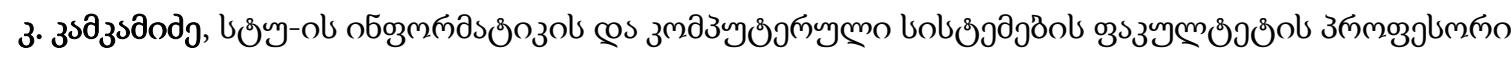

E-mail: kkamkamidze@yahoo.com

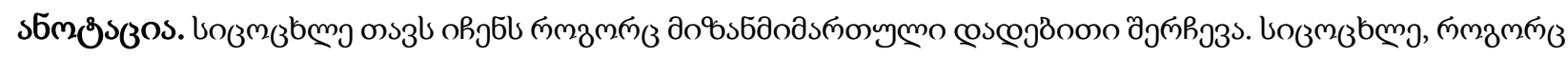

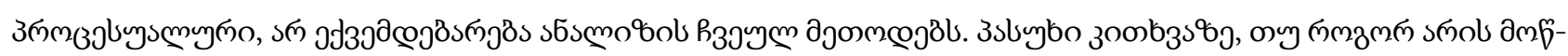

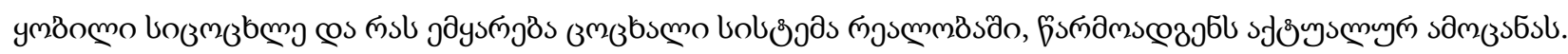

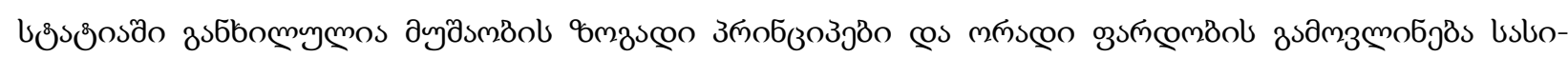

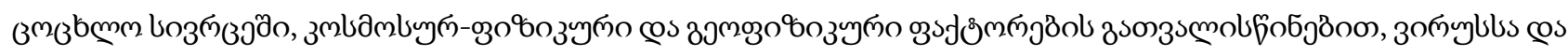

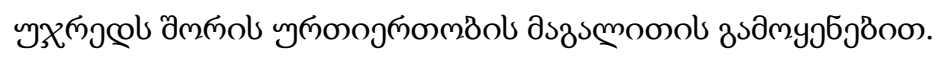

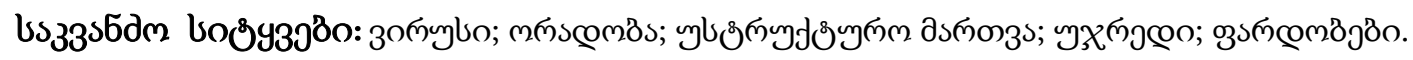




\section{UDC 513.72}

SCOPUS CODE

https://doi.org/10.36073/1512-0996-2021-1-119-135

\section{Управление в пространстве жизни}

Серго Дадунашвили Департамент электротехники и электроники, Грузинский технический университет, Грузия, 0160, Тбилиси, ул. М. Костава 75

E-mail: dadu@gtu.ge

\section{Рецензенты:}

Г. Дгебуадзе, профессор факультета энергетики и телекоммуникации, ГТУ

E-mail: project7@gtu.ge

К. Камкамидзе, профессор факультета информатики и компьютерных систем, ГТУ

E-mail: kkamkamidze@yahoo.com

\section{Аннотация.}

Жизнь проявляется как целесообразный положительный подбор. Живое, как процессуальное, не поддаётся обычным приёмам анализа. Ответ на вопрос, как устроена жизнь и на чём в реальности держится живая система, представляет актуальную задачу.

В статье рассматриваются общие принципы работы и проявления двойственных отношений в пространстве жизни, с учётом космофизических и геофизических факторов, на примере взаимоотношения вируса и клетки.

Ключевые слова: бесструктурное управление; вирус; двойственность; клетка; отношения. 